\title{
Phacomatosis Pigmentokeratotica Associated With Raynaud Phenomenon, Segmental Nevi, Hyperhidrosis, and Scoliosis
}

Faliang Ren, MD; Laura Pruitt, MD; Qi Tan, MD; Dirk M. Elston, MD

\section{PRACTICE POINTS}

- Phacomatosis pigmentokeratotica (PPK) is characterized by the coexistence of speckled lentiginous nevus and nevus sebaceous.

- Raynaud phenomenon may be an unreported association with PPK.

\section{To the Editor:}

Phacomatosis pigmentokeratotica (PPK) is a rare epidermal nevus syndrome complicated by multiple extracutaneous anomalies, including skeletal defects and neurologic anomalies. Less common associations include lateral curvature of the spine and hyperhidrosis. We present a patient with PPK and unilateral Raynaud phenomenon in addition to a segmental distribution of melanocytic nevi, hyperhidrosis, and scoliosis.

A 9-year-old girl was born with a yellow-orange alopecic plaque on the right side of the scalp (Figure 1). There also were 2 large, irregularly pigmented patches localized on the right side of the upper back and buttock. Over 3 years, numerous papular nevi developed within these pigmented patches and were diagnosed as speckled lentiginous nevi (Figure 2). In addition, numerous nevi of various sizes affected the right face, right shoulder, right arm (Figure 3), and right neck and were clearly demarcated along the midline. Several nevi also were noted within the nevus sebaceous on the right scalp. These skin lesions expanded progressively with age. At 6 years of age, she was diagnosed with hyperhidrosis of the right half of the body, which was most pronounced on the

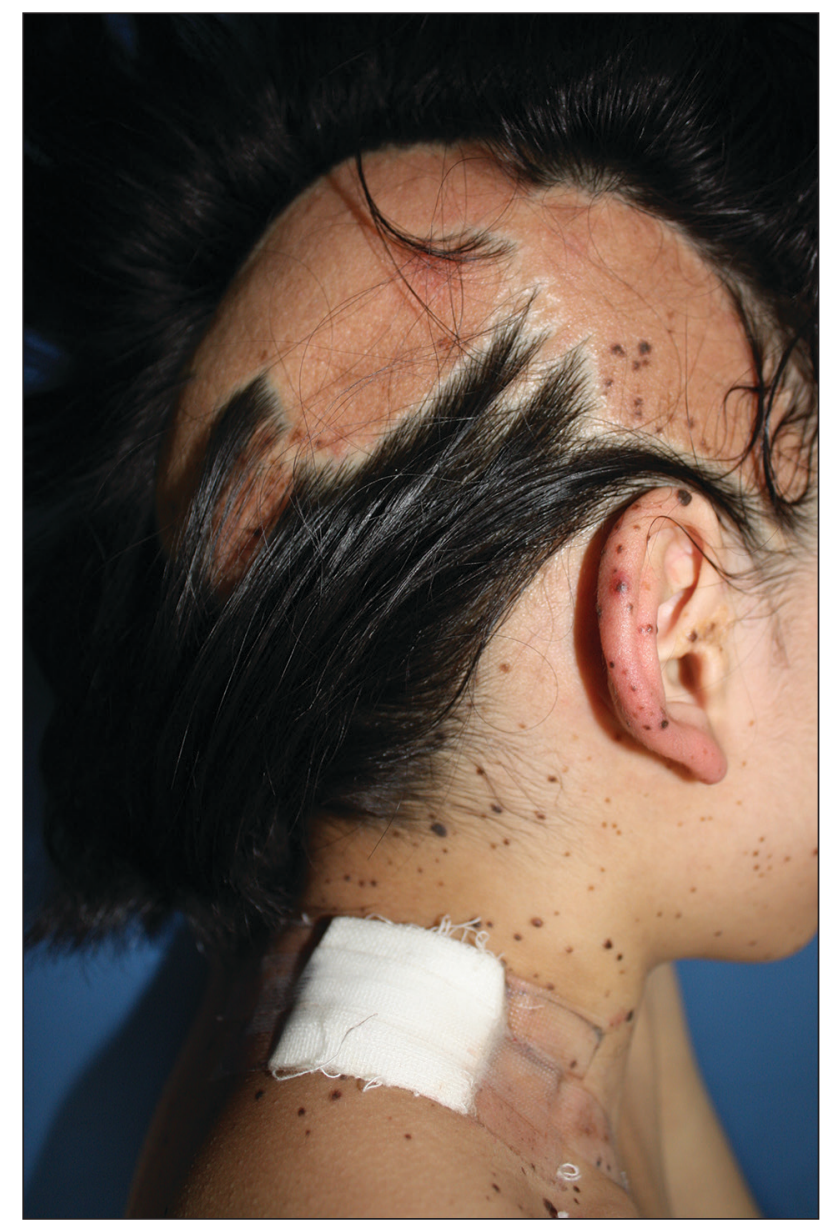

FIGURE 1. Nevus sebaceous coexisted with speckled lentiginous nevus.

Drs. Ren and Tan are from the Department of Dermatology, Children's Hospital of Chongqing Medical University, China. Drs. Pruitt and Elston are from the Department of Dermatology and Dermatologic Surgery, Medical University of South Carolina, Charleston.

The authors report no conflict of interest.

Correspondence: Qi Tan, MD, Department of Dermatology, Children's Hospital of Chongqing Medical University, 136 Zhongshan Er Rd,

Yuzhong District, Chongqing, China 400014 (dermatologyCHCMU@foxmail.com).

doi:10.12788/cutis.0279 

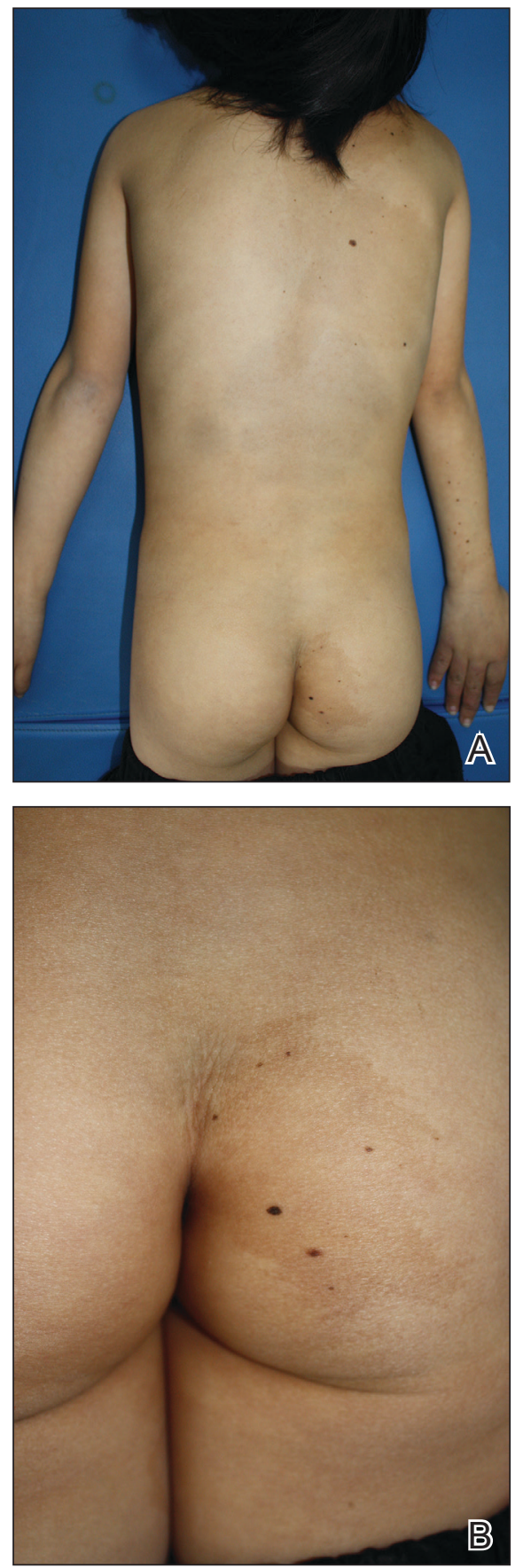

FIGURE 2. A and B, Nevus spilus on the right side of the back and buttock, respectively.

face. Raynaud phenomenon restricted to the right hand also was noted (Figure 4). Upon cold exposure, the digits become pale white, cold, and numb; then blue; and finally red. She lacked other features of connective tissue disease, and autoantibody testing was negative. She also was noted to have an abnormal lateral curvature of the spine (scoliosis). Auditory, ocular, and neurologic examinations were normal. Cranial and cerebral magnetic resonance

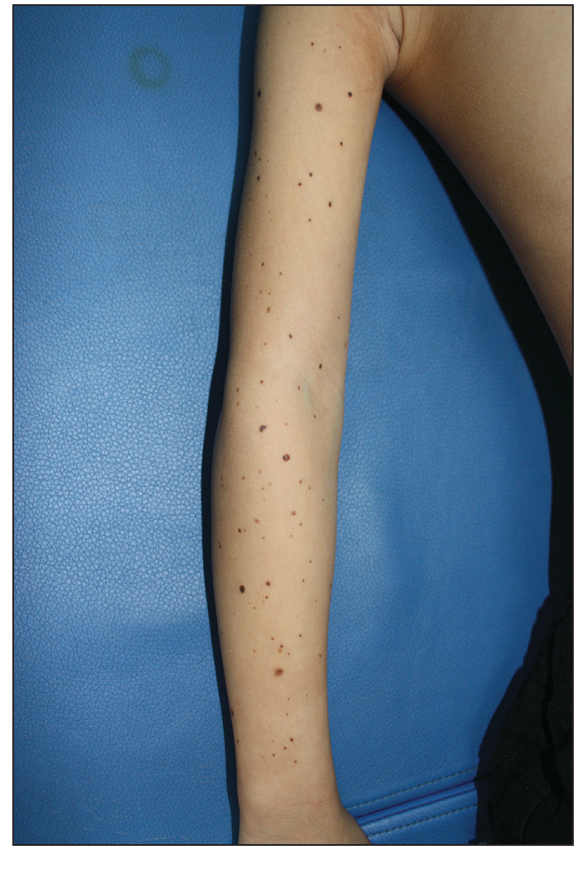

FIGURE 3. Speckled lentiginous nevi on the right arm.

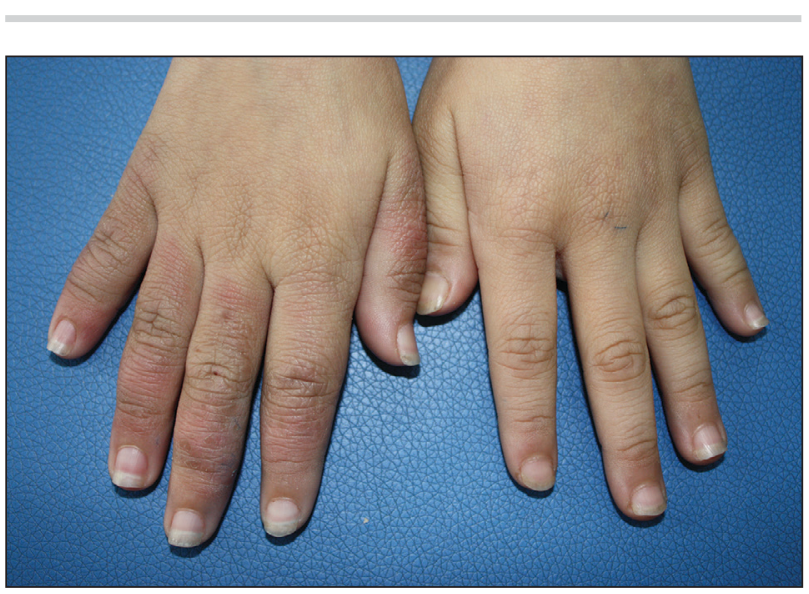

FIGURE 4. Raynaud phenomenon on the right hand.

imaging showed no central nervous system abnormalities. Her family history was negative for nevus spilus, nevus sebaceous, and neurofibromatosis. The clinical findings in our patient led to the diagnosis of PPK.

Phacomatosis pigmentokeratotica is a distinctive epidermal nevus syndrome characterized by the coexistence of a speckled lentiginous nevus, also known as a nevus spilus, and a nevus sebaceous ${ }^{1}$; PPK frequently is complicated by skeletal, ophthalmic, or neurologic abnormalities. ${ }^{2}$ Most cases reported are sporadic, and a postzygotic mosaic HRas proto-oncogene, GTPase, HRAS, mutation has been demonstrated in some patients and may contribute to the phenotype of PPK.,

Other anomalies have included ichthyosislike diffuse hyperkeratosis, laxity of the hands, pelvic hypoplasia, glaucoma, psychomotor retardation, and hypophosphatemic 
rickets. These patients also should be monitored for the development of malignant neoplasms within the nevus sebaceous. ${ }^{5}$ Segmental hyperhidrosis may be seen in association with the nevus spilus component. ${ }^{2}$

Raynaud phenomenon involving only the right hand was a unique finding in our patient. In 3 years of followup, our patient developed no evidence of connective tissue disease or other systemic illness. We speculate that Raynaud phenomenon of the right hand along with hyperhidrosis of the right side of the body could be a result of dysfunction of the autonomic nervous system. We propose that Raynaud phenomenon represents an unusual manifestation of PPK and may broaden the spectrum of extracutaneous anomalies associated with the disease. The finding of segmental nevi outside of the confines of the nevus spilus was another unusual manifestation of mosaicism.

\section{REFERENCES}

1. Happle R, Hoffmann R, Restano L, et al. Phacomatosis pigmentokeratotica: a melanocytic-epidermal twin nevus syndrome. Am J Med Genet. 1996;65:363-365.

2. Happle R. The group of epidermal nevus syndromes part I. well defined phenotypes. J Am Acad Dermatol. 2010;63:1-22, 23-24.

3. Groesser L, Herschberger E, Sagrera A, et al. Phacomatosis pigmentokeratotica is caused by a postzygotic HRAS mutation in a multipotent progenitor cell. J Invest Dermatol. 2013;133:1998-2003.

4. Martin RJ, Arefi M, Splitt M, et al. Phacomatosis pigmentokeratotica and precocious puberty associated with HRAS mutation. Br J Dermatol. 2018;178:289-291.

5. Chu GY, Wu CY. Phacomatosis pigmentokeratotica: a follow-up report with fatal outcome. Acta Derm Venereol. 2014;94:467-468. 